\title{
Stokes shift in quantum wells: trapping versus thermalization
}

\author{
A. Polimeni, A. Patanè, M. Grassi Alessi, and M. Capizzi \\ Istituto Nazionale di Fisica della Materia - Dipartimento di Fisica, Universitá di Roma "La \\ Sapienza", P.le A. Moro 2, I-00185 Roma, Italy \\ F. Martelli \\ Fondazione Ugo Bordoni, via B. Castiglione 59, I-00142 Roma, Italy \\ A. Bosacchi and S. Franchi \\ Consiglio Nazionale delle Ricerche, Istituto Materiali Speciali per l'Elettronica e Magnetismo, via \\ Chiavari 18/A, I-43100 Parma
}

(October 13, 2018)

\begin{abstract}
Low temperature photoluminescence and photoluminescence excitation measurements have been performed in a set of InGaAs/GaAs samples with different indium molar fraction, well width, growth conditions and post-growth treatment. This has allowed to change in a controlled way the degree and source of disorder in the samples, thus resulting in an excitonic absorption linewidth varying between 1 and $18 \mathrm{meV}$, and an ensueing Stokes shift changing between zero and $8 \mathrm{meV}$. The conditions of validity of two different models relating the Stokes shift to the linewidth broadening have been established in terms of different regimes of disorder and temperature. A continuous transition between those regimes has been demonstrated.

PACS numbers: 71.55.Jv, 78.55.Cr, 73.20.Dx
\end{abstract}

Typeset using REVTEX 
The full width at half maximum (FWHM) of the heavy-hole free exciton (HHFE) recombination and the Stokes shift SS, i.e., the difference between the exciton peak energy as determined by photoluminescence (PL) and absorption measurements, are commonly considered indicative of the sample quality. However, the origin of the Stokes shift is still argument of debate, although it has been the subject of a number of experimental and theoretical works. [1] In the pioneering works in $\mathrm{GaAs} / \mathrm{Al}_{x} \mathrm{Ga}_{1-x} \mathrm{As}$ single quantum wells (QWs), [1],2] the $S S$ was attributed to trapping of excitons at interface defects with a contribution to the interface potential described by a gaussian function. Assuming a lack of exciton thermalization, i.e., a recombination of excitons at the defect sites where they are first trapped, the $S S$ is determined by the distribution of the binding energy of excitons to the defects. The vanishing of $S S$ for increasing temperature has been attributed to an increased detrapping of excitons. In later works, the main cause of exciton trapping to a disordered potential has been identified in the interface roughness produced by an inhomogeneous thickness of the QWs. [3:4] In other theoretical models, a major emphasis has been given, instead, to thermal effects on carrier migration, [0] the carrier thermalization [6] or the thermodynamical balance between excitons and radiation. [7] We will briefly resume in the following those models which lead to an analytical relationship between $S S$ and the value $W$ of FWHM of the absorption line and thus allow a comparison with experimental data.

In the model of Fang Yang et al, [3] the probability distribution of the exciton energy in the QW-plane (x,y) is assumed to be a function $G(x, y)$, which determines the shape of the absorption band. Fluctuations in the QW thickness, with a gaussian distribution due to the diffusion process during the QW growth, [4 are assumed to be the only source of randomness in the exciton energy distribution. If this topographical disorder extends over a scale much greater than the carrier diffusion length, carriers relax into local minima before recombining as excitons. Therefore, the PL reflects the distribution of local minima in the exciton-energy distribution and a $S S$ ensues, with

$$
S S=0.55 \mathrm{~W} .
$$


A linear relationship is found, indeed, between $S S$ and $W$ as obtained in several III-V and II-VI compounds, with a proportionality factor equal to 0.6. It is worth noticing that the values of $W$ range from a few meV to about $100 \mathrm{meV}$, suggestive of a medium to high degree of disorder. A similar linear behavior, although affected by a sizeable scattering in the data, has been also obtained in the case of symmetrically strained (GaIn)As/Ga(PAs) superlattices for $W$ varying between 6 and $10 \mathrm{meV}$. [8]

If thermal effects in the exciton diffusion are added to a gaussian disorder, only numerical estimates can be carried out. [5] A linear relationship between $S S$ and the PL linewidth is found to hold in the low temperature limit, with a slope smaller (0.3) than that found in absence of thermal effects. No comparison with experimental results is provided by the authors.

In the model of Gurioli and coworkers, [6] the emphasis is further shifted toward the role of thermal effects. Excitons are assumed to be in thermal quasiequilibrium, with a distribution characterized by an effective carrier temperature $T_{c}$ greater or equal to that of the lattice. The Stokes shift is then related to the degree of carrier thermalization, as measured by the ratio between the carrier thermal energy $\mathrm{k}_{B} T_{c}$ and the inhomogeneous broadening of the exciton-band. Therefore, $S S$ becomes vanishingly small whenever that ratio is much greater than unity (namely, when a high temperature allows to establish a thermal equilibrium). Otherwise, the high energy side of the exciton distribution function is depressed in PL because of population effects, and a sizeable $S S$ results. Provided that the disorder responsible for the exciton broadening is described by a gaussian function, the model results into

$$
S S=0.18 W^{2} / k_{B} T_{c}
$$

in quite good agreement with experimental data in AlGaAs/GaAs QWs where $W$ is of the order of a few meV or less. Therefore, both models described above account for the origin of the Stokes shift if applied to systems which largely differ in the degree (and nature) of disorder. 
Recently, our group has studied the effects of structural disorder on the photoluminescence of strained InGaAs/GaAs quantum wells grown by molecular beam epitaxy (MBE) [9.10] and differing in the indium molar fraction $x$ and/or well width $L$. A detailed analysis of the PL lineshape for increasing $x$ and $L$ has evidentiated the role and weight of alloy disorder and well-width fluctuations - namely, of the main mechanisms responsible for the spectral broadening of HHFE recombination. [9] Potential fluctuations give also rise to localization of excitons, most likely at the interface. [10] In the present work, we will compare the two analytical models on the origin of $S S$ previously resumed with experimental results in those strained InGaAs/GaAs heterostructures. In this case, the HHFE peak energy and absorption linewidth are obtained from PL excitation (PLE) measures because of the low absorbance of quantum wells. [11 By tuning the amount and source of disorder in the InGaAs/GaAs QWs, we will show that both the topographical model [3, [4] and the quasiequilibrium model [6] hold in this system. Those models, indeed, well describe the experimental data in the two opposite limits of large or small $W$ (disorder), respectively, with a crossover between the two regimes for $W \sim 6 \mathrm{meV}$. Moreover, for increasing lattice temperatures data turn out to be well described by the quasiequilibrium model also in cases where the topographical model holds at low temperature.

The samples used for this work are $\operatorname{In}_{x} \mathrm{Ga}_{1-x}$ As/GaAs single QWs grown on GaAs(100) substrates, with $x=0.09,0.10,0.19,0.80$, and 1.0 and different well widths (see Table I). Only single QWs have been considered in order to eliminate possible inter-well fluctuations. The $x=0.09,0.19$ and $x=1.0$ samples, the same used in Ref. 9, have been grown by MBE in a Varian GenII machine at $520{ }^{\circ} \mathrm{C}$; further details on the $x=1.0 \mathrm{InAs} / \mathrm{GaAs}$ sample-growth are given elsewhere. [12] Samples with $x=0.10(x=0.80)$ were grown in a different MBE machine at $360{ }^{\circ} \mathrm{C}\left(460{ }^{\circ} \mathrm{C}\right)$, in order to reduce both In segregation [13] and In surface migration. The same applies to samples irradiated with deuterium by a Kauffman source, as discussed in the following. The PL measurements have been performed by exciting the samples with an $\mathrm{Ar}^{+}$laser. A Ti:Sapphire laser has been used as light source in the PLE measurements. In both cases the luminescence was dispersed through a $65-\mathrm{cm}$ 
double-grating monochromator and detected by standard photon counting tecniques. The samples were held at temperatures ranging between $5 \mathrm{~K}$ and $70 \mathrm{~K}$.

In Figure 1, we report typical PL and PLE spectra showing the HHFE transition for different indium concentrations (the values of $W$ and of $S S$ for all samples investigated are given in Table I). For ease of comparison, the PL and PLE spectra have been reported by taking for each sample the zero of the energy at the PLE peak. The Stokes shift and the recombination and absorption broadenings are clearly correlated, both increasing from the top to the bottom of the figure. The shoulder on the low energy side in the spectrum of the sample shown in Fig. 1(a) is due to an exciton bound to interfacial disorder. 10 The peak at about $10 \mathrm{meV}$ above the HHFE in the sample reported in Fig. 1(b) is due to the partially-forbidden transition e1-hh3, usually observed in wide QWs. [14] In Fig. 1(c), the low- and high-energy bands in the PL spectrum are due to the recombination of the carriers in the quantum dots formed during the growth and in the flat InAs QW, respectively. [15] In the InAs/GaAs QWs, both PL and PLE measures have been performed on deuterated samples, where a giant increase in the luminescence efficiency makes measures easier. [12] It is worth noting that the diffusion of deuterium, besides increasing the PL signal, produces an increased degree of disorder, as evidentiated by a sizeable brodeaning of the luminescence and absorption recombination bands (not shown here).

In Figure 2, we plot the values of $S S$ as a function of $W$ as measured in the PLE spectra for the different samples. The data corresponding to high values of $S S$, namely, where the disorder dominates the PL spectra, fall on a straight line as expected from the model introduced in Refs. 3 and 4. Some scattering in the data appears instead for low values of $W$, as reported also in Ref. 8 for the same range of $W$ s. The full line reported in the figure for $W \geq 6 \mathrm{meV}$ is a best fit of a linear function to the data $(S S=-0.6+0.48 \mathrm{~W})$. The linear behavior, as well as the slope, are in good agreement with the relation found in Ref. 3, although the fit accounts for null values of $S S$ at finite values of $W$, a feature not predicted by the model. [16]

Let us now give a closer inspection to the region of small $W(\leq 6.0 \mathrm{meV}$, or $S S \leq 2.0$ 
meV), where the linear extrapolation from the high disorder regime (dashed line in Fig. 2) gives a poor fit to the data and the validity of the linear model has been questioned. [6] In Figure 3, we compare the experimental results with the Eq. (2) of the thermalization model, where $T_{c}$ has been estimated from the high-energy tail of the PL spectra. The thermalization model well accounts for the experimental results in this region of low $W$ values. A quadratic relationship between $S S$ and $W$ does not hold, instead, for the whole set of data, as shown in the inset where the model clearly deviates from the experimental data for $W \geq 6 \mathrm{meV}$.

As from Figures 2 and 3, our data agree better with one model or the other according to the values of $S S$ and FWHM, namely, to the sample "quality". This is a consequence of the different physical mechanisms introduced in the two models to explain the red shift with respect to the absorption peak of the HHFE recombination line. If one assumes that the thermalization is the predominant mechanism, all the energy levels contributing to the absorption are in thermal equilibrium and the carriers diffuse to the lowest energy state available before they recombine. As a matter of fact, the experimental data are explained by the thermalization model in the samples where the thermal energy is about equal to the FWHM (e. g., $x=0.19$ and $x=0.09$ for small $L$, see Table I) and a stronger communication between the broadened excitonic levels is expected. On the other hand, the carrier diffusion becomes less effective whenever the sample inhomogeneities give rise to local energy minima and to potential fluctuations large with respect to the carrier thermal energy. In this case, if hopping processes between local minima are ignored (see discussion in Ref. 17), the different energy levels do not interact with each other and the PL is ruled by the distribution of heights of local minima, as stated by Yang and coworkers. In the InAs/GaAs QWs, the fluctuations of $L$ (at least one ML) is quite large with respect to its mean value (2 ML or less). Moreover, quantum dots self-aggregate at the interface [15] thus giving rise to a further source of disorder. In this case, the thermal energy turns out to be quite lower than $W$ thus satisfying the basic assumption of deep local minima associated with a strong disorder.

Let us now comment the limit of validity of the topographical model. In Ref. 9, it has been shown that alloy and interface disorder account for the PL linewidth. The lateral 
extent of the potential fluctuations at the interface has been estimated in about $2 \mathrm{~nm}$, one order of magnitude smaller than the exciton Bohr radius $(\sim 20 \mathrm{~nm})$. This results in a single, usually narrow HHFE recombination line. A very similar picture has been given recently in a theoretical paper. [18] Broader HHFE recombination lines can be expected when growing the samples at a lower temperature, because a lower In surface migration increases the interface- and alloy-disorder. The samples grown at $360{ }^{\circ} \mathrm{C}(\mathrm{x}=0.10)$ have, indeed, a larger linewidth with respect to samples [9] grown at $520{ }^{\circ} \mathrm{C}$, especially for wide $L$ (this last feature suggests that the low temperature growth affects the alloy disorder more than the interface roughness). Moreover, also a deuterium diffusion into the samples results in an increased disorder, most likely on a short-scale. The disorder accounting for the FWHM in InGaAs/GaAs QWs is therefore always on a scale length smaller than the exciton dimension. In Refs. 3 and 4, it is stated that the fluctuations in the potential are dominated by variations of the effective width over length scale greater than the exciton size, whereas variations of the well width over smaller dimensions are smeared out by the finite extension of the exciton wavefunction. However, this does not prevent that model from holding in the present case. In fact, although individual excitons feel potential fluctuations averaged over a scale length smaller than their radius, the mean potential value found on the exciton radius fluctuates over the whole sample. [9] Flat regions on the scale of the exciton size, which would give rise to multiple PL peaks, [19 not present in our samples, are therefore not a mandatory feature of the topographical model.

A further support to the picture of a continuous transition between the quasiequilibrium and the topographical disorder model is provided by the dependence on temperature of the Stokes shift reported in Fig. 4(a) for the InAs/GaAs sample with $L=1.2$ ML. At $5 \mathrm{~K}$, this sample has a PLE linewidth equal to $13 \mathrm{meV}$ (see Table I) and belongs, therefore, to the samples where the topographical model better works. However, when the temperature increases from $5 \mathrm{~K}$ to $70 \mathrm{~K}, S S$ decreases from 6 to $4.1 \mathrm{meV}$, a behavior which cannot be explained by the topographical model. On the other hand, an increasing value of the ratio $\mathrm{k}_{B} T_{c} / W$ between the thermal energy and the disorder may convert a regime where 
the local disorder dominates the spectra into a regime where the carrier thermalization is predominant. This is, indeed, the case of the present sample, as shown in Fig. 4(b) where the difference $\triangle S S$ between the measured value of the Stokes shift and that predicted by the quasiequilibrium model, renormalized with respect to the experimental value, is reported vs $\mathrm{k}_{B} T_{c} / W$. As expected, $\triangle S S / S S$ decreases for increasing $\mathrm{k}_{B} T_{c} / W$.

In conclusion, photoluminescence and photoluminescence excitation measurements in InGaAs/GaAs quantum wells whose morphology has been gradually varied by changing the indium concentration, the well width, the growth conditions and/or post-growth treatments, have shown that different functional relationships between the $S S$ and the FWHM hold for different degree of interplay between thermalization and disorder. In samples with low In-concentration, the thermalization process between the inhomogeneously broadened absorption band dominates. When the FWHM is large enough to make thermalization unlikely, the topographical model proposed by Yang and coworkers applies. Measurements as a function of temperature have also confirmed that those two regimes can be continuously converted one into the other. 


\section{TABLES}

TABLE I. Values of the $S S$ and of the linewidth of PLE for different indium concentration $x$, well width $L$ and growth temperature $T_{G}$

\begin{tabular}{|c|c|c|c|c|}
\hline $\begin{array}{c}L \\
(\mathrm{~nm})\end{array}$ & $x$ & $\begin{array}{c}T_{G} \\
\left({ }^{\circ} \mathrm{C}\right)\end{array}$ & $\begin{array}{c}S S \\
(\mathrm{meV})\end{array}$ & $\begin{array}{c}W \\
(\mathrm{meV})\end{array}$ \\
\hline 5.0 & 0.09 & 520 & 0.0 & 1.2 \\
\hline 5.0 & 0.10 & 360 & 0.8 & 2.8 \\
\hline 10.0 & 0.10 & 360 & 0.7 & 3.6 \\
\hline 20.0 & 0.10 & 360 & 1.8 & 6.0 \\
\hline 1.5 & 0.19 & 520 & 0.3 & 1.4 \\
\hline 2.0 & 0.19 & 520 & 0.7 & 1.6 \\
\hline 3.0 & 0.19 & 520 & 1.4 & 3.8 \\
\hline 4.0 & 0.19 & 520 & 1.6 & 4.5 \\
\hline 7.0 & 0.19 & 520 & 1.3 & 3.1 \\
\hline \multicolumn{5}{|l|}{ (ML) } \\
\hline 2.0 & 0.80 & 460 & 2.3 & 6.0 \\
\hline 0.6 & 1.0 & 420 & 2.9 & 7.0 \\
\hline 0.8 & 1.0 & 420 & 3.9 & 9.2 \\
\hline 1.0 & 1.0 & 420 & 4.7 & 10.4 \\
\hline 1.2 & 1.0 & 420 & 6.0 & 13.0 \\
\hline 1.6 & 1.0 & 420 & 7.8 & 18.0 \\
\hline
\end{tabular}




\section{REFERENCES}

[1] G. Bastard, C. Delalande, M. H. Meynadier, P. M. Frijlink, and M. Voos, Phys. Rev. B 29, 7042 (1984).

[2] C. Delalande, M. H. Meynadier, and M. Voos, Phys. Rev. B 31, 2497 (1985).

[3] F. Yang, M. Wilkinson, E. J. Austin, and K. P. O’Donnell, Phys. Rev. Lett. 70, 323 (1993).

[4] M. Wilkinson, F. Yang, E. J. Austin, and K. P. O’Donnell, J. Phys. Condens. Matter 4, 8863 (1992).

[5] Er-Xuan Ping and Vikram Dalal, J. of Appl. Phys. 74, 5349 (1993).

[6] M. Gurioli, A. Vinattieri, J. Martinez-Pastor, and M. Colocci, Phys. Rev. B 50, 11817 (1994).

[7] J. Humlicek, E. Schmidt, L. Bocnek, R. Svehla, and K. Ploog, Phys. Rev. B 48, 5241 (1993).

[8] S. Lutgen, T. F. Albrecht, T. Marschner, W. Stolz, and E. O. Goebel, Solid-State Electronics, 37, 905 (1994).

[9] A. Patanè, A. Polimeni, M. Capizzi, and F. Martelli, Phys. Rev. B 52, 2784 (1995).

[10] F. Martelli, A. Polimeni, A. Patanè, M. Capizzi, P. Borri, M. Gurioli, M. Colocci, A. Bosacchi, and S. Franchi, Phys. Rev. B 53, 7421 (1996).

[11] This equivalence is not strictly true because the PLE lineshape is also influenced by carrier dynamics. However, the distortion in the FWHM introduced by this effect can be assumed to be within the present experimental uncertainty in $W$.

[12] A. Polimeni, D. Marangio, M. Capizzi, A. Frova, and F. Martelli, Appl. Phys. Lett. 65, 1254 (1994). 
[13] A. Bosacchi, F. Colonna, S. Franchi, P. Pascarella, P. Allegri, and A. Avanzini, J. Crystal Growth 150, 185 (1995).

[14] J. D. Lambkin, L. K. Howard, and M. T. Emeny, Phys. Rev. B 42, 1738 (1990).

[15] A. Polimeni, A. Patanè, M. Capizzi, F. Martelli, L. Nasi, and G. Salviati, Phys. Rev. B 53, R4213 (1996).

[16] A zero value for $S S$ has been found in all samples of a set having $x=0.09$ and grown at $520{ }^{\circ} \mathrm{C}$. The largest FWHM of the PLE in this set of samples $(1.2 \mathrm{meV})$ is reported in Fig. 1 (see Ref. 9 for further informations on PL).

[17] J. A. Kash, Phys. Rev. Lett. 71, 1286 (1993); F. Yang, M. Wilkinson, E. J. Austin, and K. P. O’Donnell, ibid. 71, 1287 (1993).

[18] Al. L. Efros, C. Wetzel, and J. M. Worlock, Phys. Rev. B 52, 8384 (1995).

[19] see, e.g., R.F. Kopf, E.F. Schubert, T.D. Harris, and R.S. Becker, Appl. Phys. Lett. 58, 631 (1991). 


\section{FIGURES}

FIG. 1. Low temperature photoluminescence (dashed lines) and photoluminescence excitation spectra (full lines) of the heavy-hole free exciton in three InGaAs/GaAs samples with different well width and indium molar fraction. The Stokes shift increases from the top to the bottom of the figure, as well as the HHFE recombination and absorption linewidths. For ease of comparison, a common zero of the energy has been taken at the PLE peak of HHFE (at 1.470, 1.400, and 1.471 $\mathrm{eV}$, from top to bottom of the figure).

FIG. 2. The Stokes shift as a function of the FWHM of the HHFE band measured in PLE for all the investigated samples. The full line, $S S=-0.6+0.48 \mathrm{~W}$, has been obtained by a best fit to the data for $W \geq 6 \mathrm{meV}$. The dashed line extrapolates the best fit to $W<6 \mathrm{meV}$. Typical error bars are given for the sample with $W=10.4 \mathrm{meV}$.

FIG. 3. The product of the thermal carrier energy $\mathrm{k}_{B} T_{c}$ and the Stokes shift vs $W^{2}$ for FWHMs of the HHFE band measured in PLE smaller than $6 \mathrm{meV}$ (data reported already in Fig. 1). The

full line, $S S \dot{k}_{B} T_{c}=0.18 W^{2}$, gives the behavior predicted by the quasiequilibrium model of Ref. 6. In the inset, the comparison between the data and the same model is extended to the full set of samples.

FIG. 4. (a) The Stokes shift as a function of lattice temperature, $T$, for the InAs/GaAs sample with $L=1.2$ ML. (b) Ratio between the deviation of the experimental value of $S S$ from that predicted by the quasiequilibrium model and $S S$ itself as a function of $\mathrm{k}_{B} T_{c} / W . T_{c}$ is the carrier temperature as determined by an exponential fit to the high energy tail of the recombination band. The dashed lines are guides to the eye. 